\title{
Multislice computed tomography evaluation of primary abdominal fat necrosis: a rare cause of acute abdominal pain
}

\author{
Wael H. Kamr ${ }^{A, B, C, D, E, F}$, Saher E. Taman ${ }^{A, B, D, E, F}$, Ahmed I. Tawfik ${ }^{A, B, D, E, F}$ \\ Department of Radiology, Faculty of Medicine, Mansoura University, Mansoura, Egypt
}

\section{Abstract}

\begin{abstract}
Purpose: Abdominal fat necrosis is a rare cause of abdominal acute pain, classified into primary or secondary according to the cause. Primary fat necrosis includes epiploic appendagitis or idiopathic infarction of the greater omentum. This retrospective study focuses on multislice computed tomography (MSCT) findings and diagnosis of primary abdominal fat necrosis as a cause of acute abdomen.

Material and methods: This was a retrospective study with 20 patients included, presented to emergency room with acute abdominal pain diagnosed as primary fat necrosis. Retrospective evaluation was made of the patients' clinical data, presentation, CT studies done at the acute stage, and their primary and final diagnosis.

Results: Twenty patients (eight male and 12 female, mean age 45 years, age range 20-70 years) diagnosed with abdominal fat necrosis (primary omental infarct) on CT imaging between October 2014 and June 2018 were evaluated. Clinically, five patients were suspected to be cholecystitis, eight patients as appendicitis, and four patients as diverticulitis. In addition, three patients had renal colic and were suspected to have ureteric stones; they showed suspected areas of abnormal fat density in non-contrast CT of the urinary tract. Idiopathic omental infarctions were detected in 13 patients on CT; all were on the right side. Laparoscopic excision was done for all. The other seven patients had epiploic appendagitis, seen on the left side, treated with conservative management.
\end{abstract}

Conclusions: Primary fat necrosis, although rare, can be presented as acute abdomen. MSCT is the main diagnostic tool for diagnosis of omental infraction and differentiation between other causes of acute abdomen.

Key words: acute abdomen, MSCT, epiploic appendagitis, abdominal fat necrosis.

\section{Introduction}

Abdominal fat necrosis (AFN) is a rare cause of abdominal pain and may have a clinical manifestation of acute abdomen. However, it may be asymptomatic and accompany other pathophysiological processes. There have been about 300 cases of omental infarction reported since the first case reported in 1896 by Bush [1,2].

Abdominal fat necrosis is classified as primary or secondary according to the cause. Primary fat necrosis includes epiploic appendagitis (EA) or idiopathic omental infarction (IOI) of the greater omentum. The latter is usually presented by right upper or lower quadrant pain that could not be differentiated clinically from acute pain induced by appendicitis or acute cholecystitis [3].

While secondary fat necrosis is related to certain causes like trauma, post-operative, pancreatitis or malignancy and can be at any site of abdomen related to the primary pathology [4].

The diagnosis of AFN is made mainly by imaging, with the modality of choice being computed tomography (CT). During CT interpretation, differentiation should be clear between the primary fat necrosis and other pathologic conditions involving the fat, like focal lipohypertrophy, pathologic fat paucity (lipodystrophies), and malig-

\section{Correspondence address:}

Dr. Wael H. Kamr, Department of Radiology, Faculty of Medicine, Mansoura University, 35511, Mansoura, Egypt, e-mail: dr.waelkamr@gmail.com

Authors' contribution:

A Study design · B Data collection · C Statistical analysis · D Data interpretation · E Manuscript preparation · F Literature search · G Funds collection 
nancies such as liposarcoma and carcinomatosis, which may mimic benign causes of fat stranding [5].

The primary goal of CT is an accurate diagnosis that helps to make a correct management decision: either surgical resection or conservative management if possible [6].

The aim of the study is to describe CT findings and features and to clarify its role in the diagnosis of primary abdominal fat necrosis.

\section{Material and methods}

Our retrospective study included 20 patients out of 1850 who presented to the emergency room with acute abdo-

Table 1. Patient characteristics

\begin{tabular}{|c|c|c|}
\hline Variable & $\begin{array}{l}\text { Idiopathic } \\
\text { omental } \\
\text { infarction }\end{array}$ & $\begin{array}{l}\text { Appendagitis } \\
\text { epiploic }\end{array}$ \\
\hline \multicolumn{3}{|l|}{ Clinical presentation } \\
\hline Nausea and vomiting & $13(65 \%)$ & $7(35 \%)$ \\
\hline Low-grade fever & $11(55 \%)$ & 0 \\
\hline Acute abdominal pain & $13(65 \%)$ & $7(35 \%)$ \\
\hline Right upper quadrant pain & $5(25 \%)$ & 0 \\
\hline Right lower quadrant pain & $8(40 \%)$ & 0 \\
\hline Left-sided pain & 0 & $7(35 \%)$ \\
\hline \multicolumn{3}{|l|}{ Provisional diagnosis } \\
\hline Cholecystitis & $5(25 \%)$ & 0 \\
\hline Appendicitis & $8(40 \%)$ & 0 \\
\hline Diverticulitis & 0 & $4(20 \%)$ \\
\hline Renal colic & 0 & $3(15 \%)$ \\
\hline \multicolumn{3}{|l|}{ CT findings } \\
\hline \multicolumn{3}{|l|}{ Site } \\
\hline Right & $13(65 \%)$ & 0 \\
\hline Left & 0 & $7(35 \%)$ \\
\hline \multicolumn{3}{|l|}{ Size } \\
\hline$<5 \mathrm{~cm}$ & 5 cases $(20 \%)$ & 7 cases $(35 \%)$ \\
\hline $5-7.5 \mathrm{~cm}$ & 8 cases $(40 \%)$ & 0 \\
\hline$<7.5 \mathrm{~cm}$ & 0 & 0 \\
\hline Relation to colonic wall & $\begin{array}{c}\text { Not abutting } \\
13(65 \%)\end{array}$ & $\begin{array}{l}\text { Abutting } \\
7(35 \%)\end{array}$ \\
\hline Hyperdense rim & $3(15 \%)$ & $7(35 \%)$ \\
\hline Sub-hepatic extension & $4(20 \%)$ & 0 \\
\hline Central hyperdense dot & 0 & $7(35 \%)$ \\
\hline Collection/abscess & 0 & 0 \\
\hline \multicolumn{3}{|l|}{ Management } \\
\hline Laparoscopic resection & $13(65 \%)$ & 0 \\
\hline Conservative & 0 & $7(35 \%)$ \\
\hline
\end{tabular}

men and in whom CT was performed between October 2014 and June 2018.

They comprised eight males and 12 females with an average age around 45 years (age range 20-70 years) diagnosed with primary AFN on CT imaging. All data of these 20 patients was collected from the PACS archiving radiology system including their age, sex, clinical presentation, and provisional diagnosis.

All the patients presented at the emergency room with acute abdominal pain and were referred to the radiology department for post-contrast CT study.

Multislice computed tomography (MSCT) examination was carried out using a SOMATOM Definition AS (64 detector) Multislice CT machine, Siemens/Germany. The protocol was as follows: $10 \mathrm{~mm}$ slice thickness and a collimation of $5.0 \mathrm{~mm}$ for the pre-contrast scan, and slice thickness $5.0 \mathrm{~mm}$ and collimation $2.5 \mathrm{~mm}$ for post-contrast scans; $120 \mathrm{kVp} ; 365 \mathrm{MAs}$ and rotation time $0.5 \mathrm{~s} ; 750 \mathrm{ml}$ of water-soluble iodinated contrast diluted to $1 \%$ used as oral contrast; $2 \mathrm{ml} / \mathrm{kg}$ of intravenous iodinated contrast injected at $2 \mathrm{ml} / \mathrm{s}$ over a period of 30-40 s. A non-contrast scan was performed followed by a post-contrast portal venous phase after $55 \mathrm{~s}$ from contrast intravenous infusion.

Many conditions can mimic omental infarction, so we relied on emergency-room patients who presented with acute abdomen and CT findings showing heterogeneous fat density lesion of the omentum. We did not include cases with postsurgical omental contusion or omental metastases.

\section{Exclusion criteria:}

- traumatic or post-surgical procedure omental contusions and retraction injuries,

- suspected or proven omental metastatic lesions,

- patients with acute pancreatitis with CT or laboratory evidence.

\section{Computed tomography evaluation}

Experienced radiologists, more than eight years post MD, evaluated all CT scans dependently and reached a consensus that the final CT interpretation of the 20 CT scans should be categorised as primary abdominal fat necrosis.

$\mathrm{CT}$ findings rely on the presence of a heterogeneous lesion centred in the omentum and containing areas of fat density, size of the lesion $<5,5-7.5$, and $>7.5 \mathrm{~cm}$, location, peripheral rim (presence/absence and regular/ irregular), relation to colon and liver, bowel wall thickening, and density pattern, size, and margins.

Descriptive statistics were calculated for patients' age, sex, history, complaint, and clinical and provisional diagnosis. CT findings were correlated with the clinical and laparoscopic findings (Table 1).

There was a clinical follow-up for all patients up to six months from the first presentation with no clinical indication for follow-up CT. 

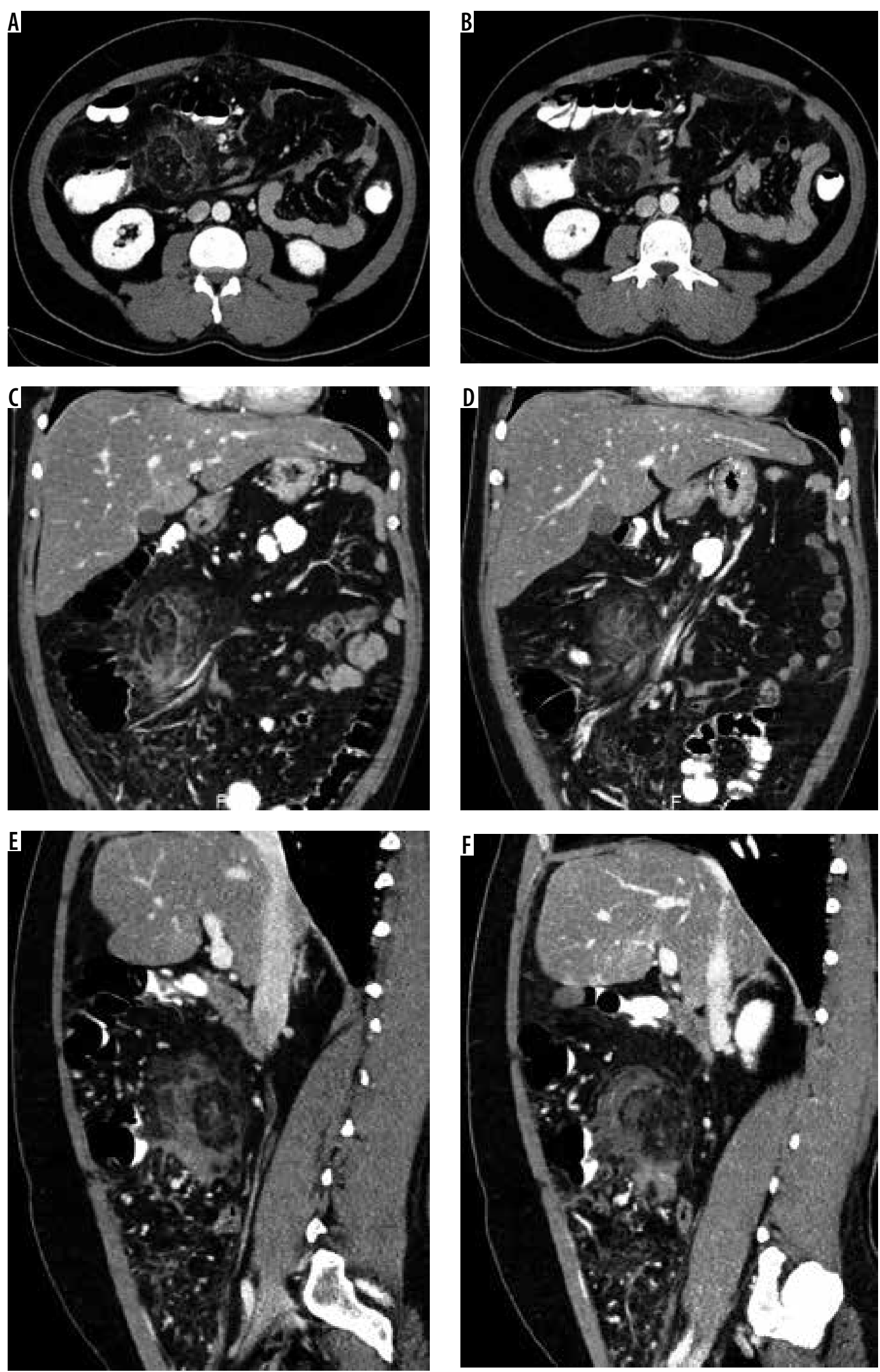

Figure 1. Omental infarction in a 40-year-old woman with right lower quadrant pain. A-B) Axial, C-D) coronal, E-F) sagittal contrast-enhanced computed tomography images shows a focal, fatty mass with soft-tissue stranding anterior to the colon - a characteristic finding of omental infarction. No other cause of abdominal pain was identified 

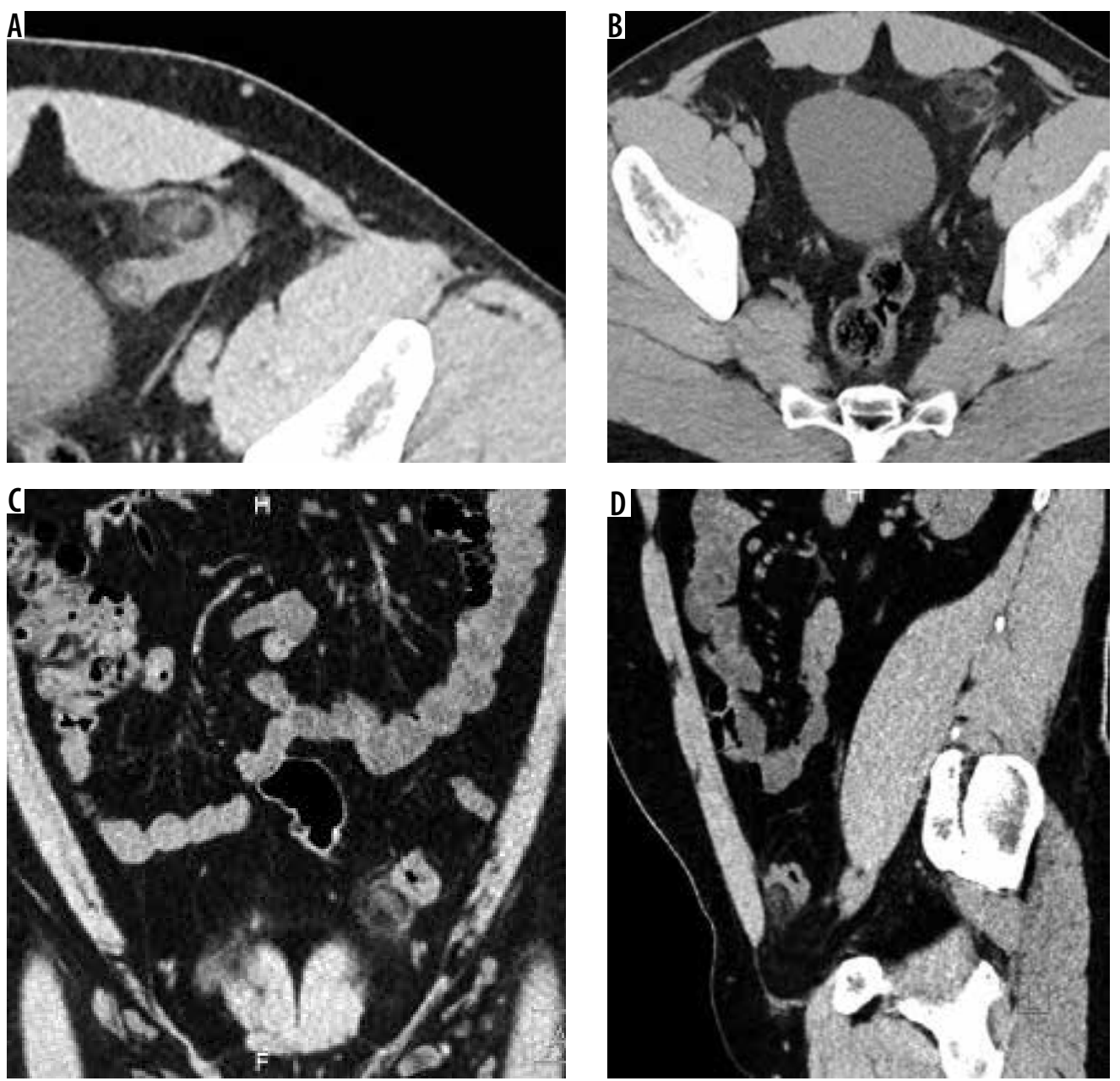

Figure 2. Epiploic appendagitis in a 32-year-old man with left lower quadrant pain. A-B) Axial and C) coronal computed tomography images showed an oval area of fat attenuation and stranding adjacent to the left colon and surrounded by a ring of soft tissue - a finding indicative of epiploic appendagitis. D) Sagittal image showing a central dot is also seen in the centre of the area of appendagitis - a finding that represents a thrombosed vein

\section{Results}

Our retrospective study included 20 patients (eight males [40\%] and 12 females [60\%], with an average age of approximately 45 years, age range 20-70 years) diagnosed with abdominal fat necrosis (omental infarct) on CT imaging between October 2014 and June 2018. All patients had primary omental fat necrosis. There were 13 patients (65\%) with idiopathic primary omental infarct (IOI), and the other seven patients (35\%) had epiploic appendagitis (EA) (Figures 1-5).

Laboratory investigations were rechecked, focusing on the white blood cell count performed within 24 hours of acute presentation. It was relatively high in 11 patients (55\%) $\left(11,000-15,000 / \mathrm{mm}^{3}\right)$, while in the remaining nine patients (45\%) the white blood cell count was within normal levels (lower than $11,000 / \mathrm{mm}^{3}$ ).

The patients were referred to perform a post-contrast CT scan based on the clinical assessment and provisional diagnosis, which was acute cholecystitis in five patients (25\%), acute appendicitis in eight patients (40\%), and diverticulitis in four patients (20\%). In addition, three pa- tients (15\%) had renal colic and were suspected to have ureteric stones. Therefore, a non-contrast CT of the urinary tract was done for them. The result was negative for stones but showed areas of abnormal fat density, so a complete contrast CT study was performed for full assessment.

According to the post-contrast MSCT findings, IOI was seen centred on the omentum, mainly along the right omental margin in 13 patients (65\%), and not abutting the colon or associated colonic wall thickening. However, there was an extension of the inflammatory reaction to the peri-cholecystic fat and sub-hepatic region in four patients (20\%). There were no detected cases with IOI located on the left side.

The other seven patients (35\%) had the lesion at the left lower quadrant, and CT findings were coping with EA, and the lesions were closely related with and abutting the colonic wall on CT.

The size of the IOI seen in 13 patients on CT, all of them were at the right side, the inflammatory lesion was 5 to $7.5 \mathrm{~cm}$ in eight patients $(40 \%)$ and smaller than $5 \mathrm{~cm}$ in five patients (25\%). No lesions were larger than $7.5 \mathrm{~cm}$. The other seven patients (35\%) had EA, all on the left side, and did not exceed $5 \mathrm{~cm}$. 

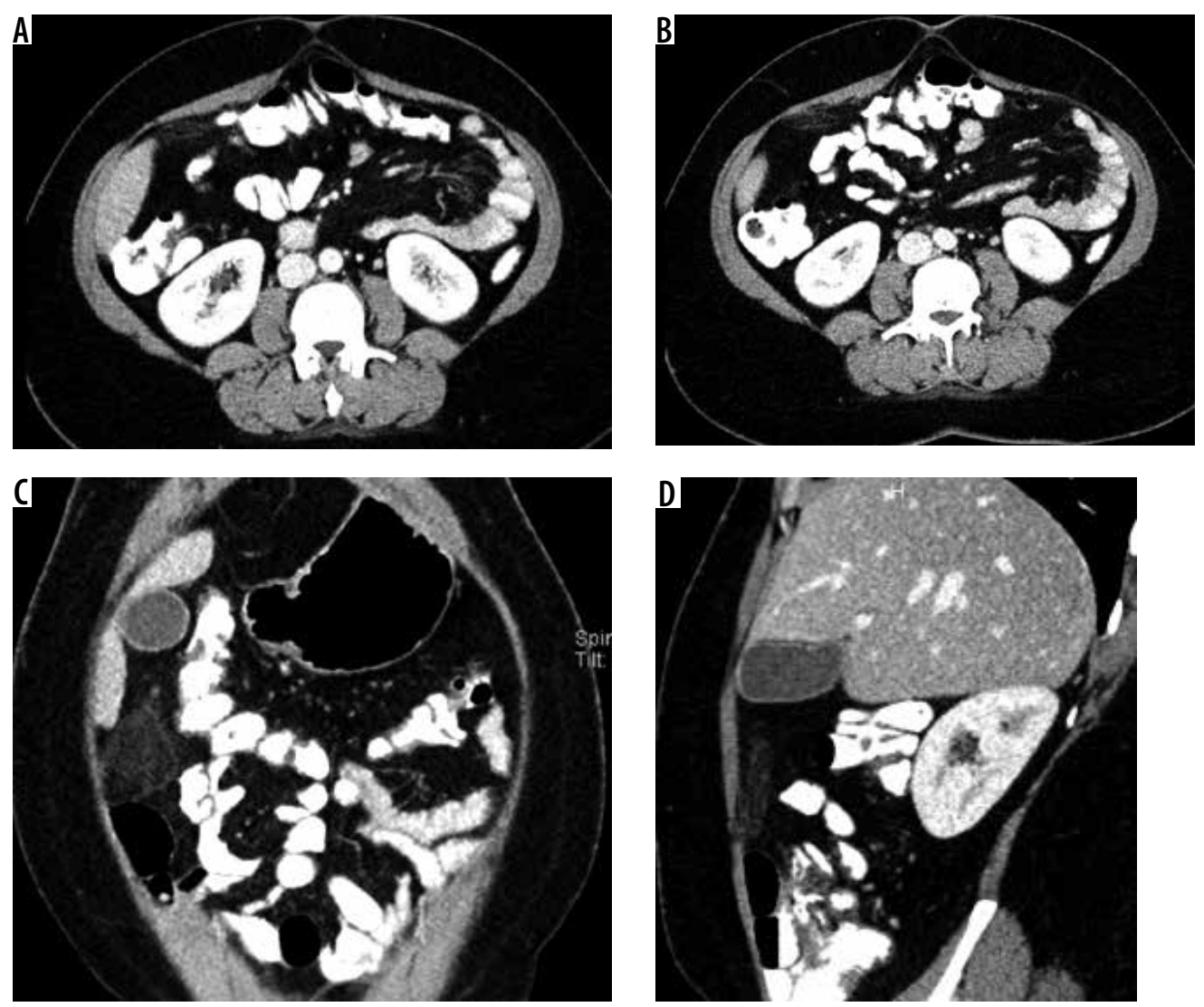

Figure 3. Omental infarction in a 66-year-old woman with right-upper quadrant pain. A-B) axial, C) coronal, and D) sagittal images showing a fatty mass adjacent to - but separate from - the ascending colon, with sub-hepatic extension a finding indicative of right-upper quadrant omental infarction

In eight patients (40\%) out of 13 with IOI, their CT showed an ill-defined, heterogeneous fat-density lesion with irregular outline and associated with soft-tissue stranding adjacent to the related colon. However, there was no colonic wall thickening detected in all cases. Smaller infarction in another five patients (25\%) showed smaller areas of abnormally increased fat density and mild haziness in the fat anterior to the colon.

The other seven patients (35\%) with EA showed in CT a small, oval area of fat attenuation surrounded by a ring of soft tissue, representing adjacent inflamed visceral peritoneum, usually anterior or anterolateral to the adjacent colon, a central hyperdense dot representing a thrombosed vein. There was no associated colonic wall thickening.

Clinical follow-up was done for all patients up to six months after the first presentation. There was no detected recurrent attack for the 13 patients with IOI and no post-operative complications. The seven patients with EA showed clinical improvement within 2-4 weeks of conservative management. There was no clinical indication for follow-up CT.

\section{Discussion}

Although acute omental infarction is rare, it can affect adults and paediatric patients [7]. However, no paediatric patients were included in our study. The patient usually presents with acute abdominal pain, commonly in the right lower quadrant. It may be associated with other symptoms like nausea, vomiting, or anorexia in addition to diarrhoea or fever. Patients with acute abdominal pain are in most cases suspected to have acute appendicitis or cholecystitis based on the clinical assessment, while the IOI is usually not suspected due to its low incidence and nonspecific clinical features $[3,4,8]$.

Our retrospective study included five patients who came to ER with acute right hypochondrial pain suspected to be cholecystitis, while another eight patients presented with right lower quadrant pain suspected to be appendicitis. On MSCT examination, they all had IOI. This shows how difficult it is to clinically assess and differentiate omental fat necrosis from other common causes of acute abdomen.

In addition, in 13 patients, IOI was seen on the right side of the abdomen because the omentum is longer and more mobile than on the left side, as reported by Almeida et al. [9]. The other seven patients had EA; all were on the left side.

It was generally noticed that 13 patients with IOI were obese and cope with Par et al. [10], who stated that obesity is one of the predisposing factors of omental infarction in addition to trauma, overexertion, surgery, sudden posture change, and congestive heart failure $[5,10]$. 

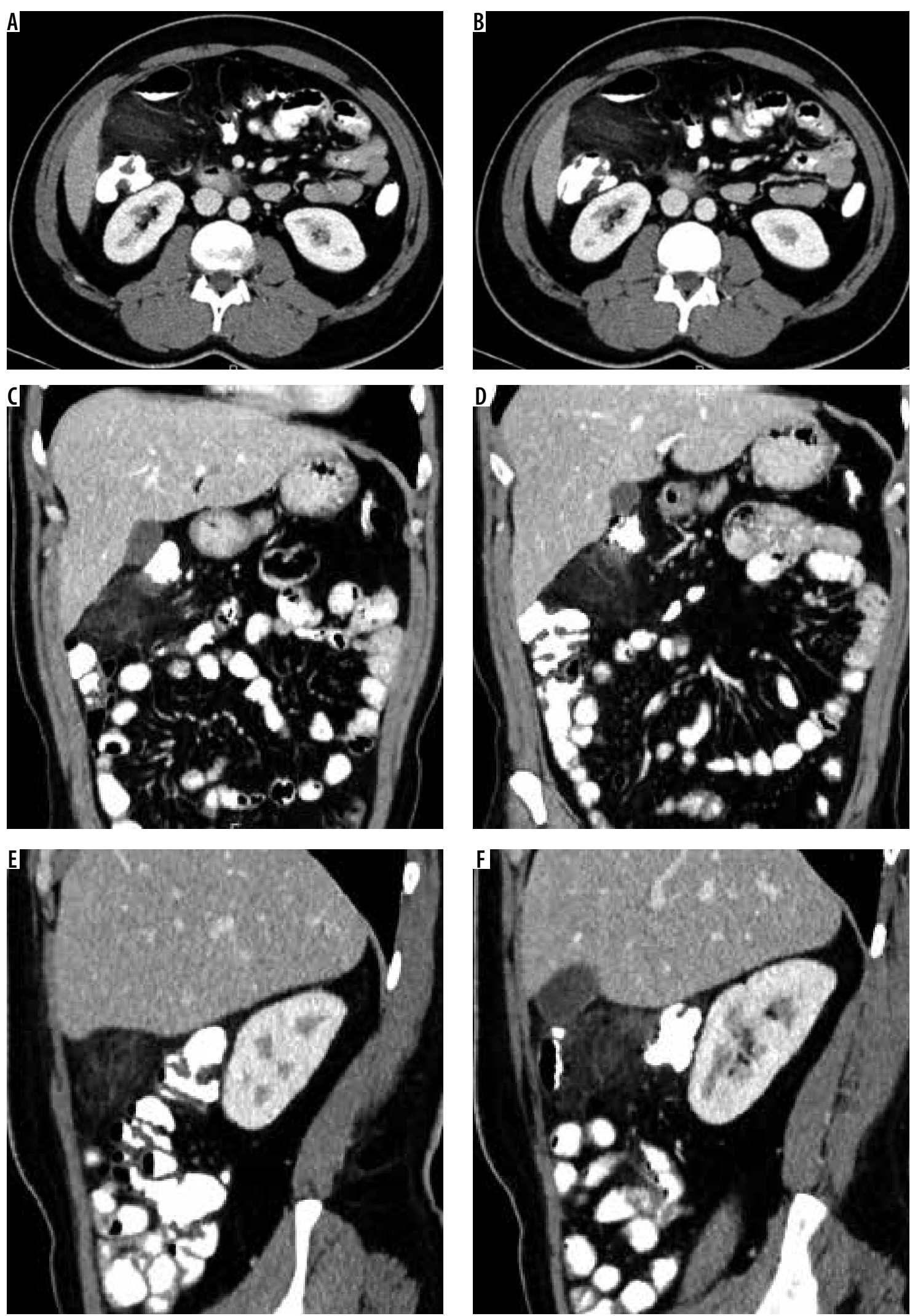

Figure 4. Omental infarction in a 56-year-old man with right-upper-quadrant pain. A-B) axial, (-D) coronal, and E-F) sagittal images showing an encapsulated fatty mass adjacent to - but separate from - the ascending colon, with sub-hepatic extension - a finding indicative of right-upper quadrant omental infarction 

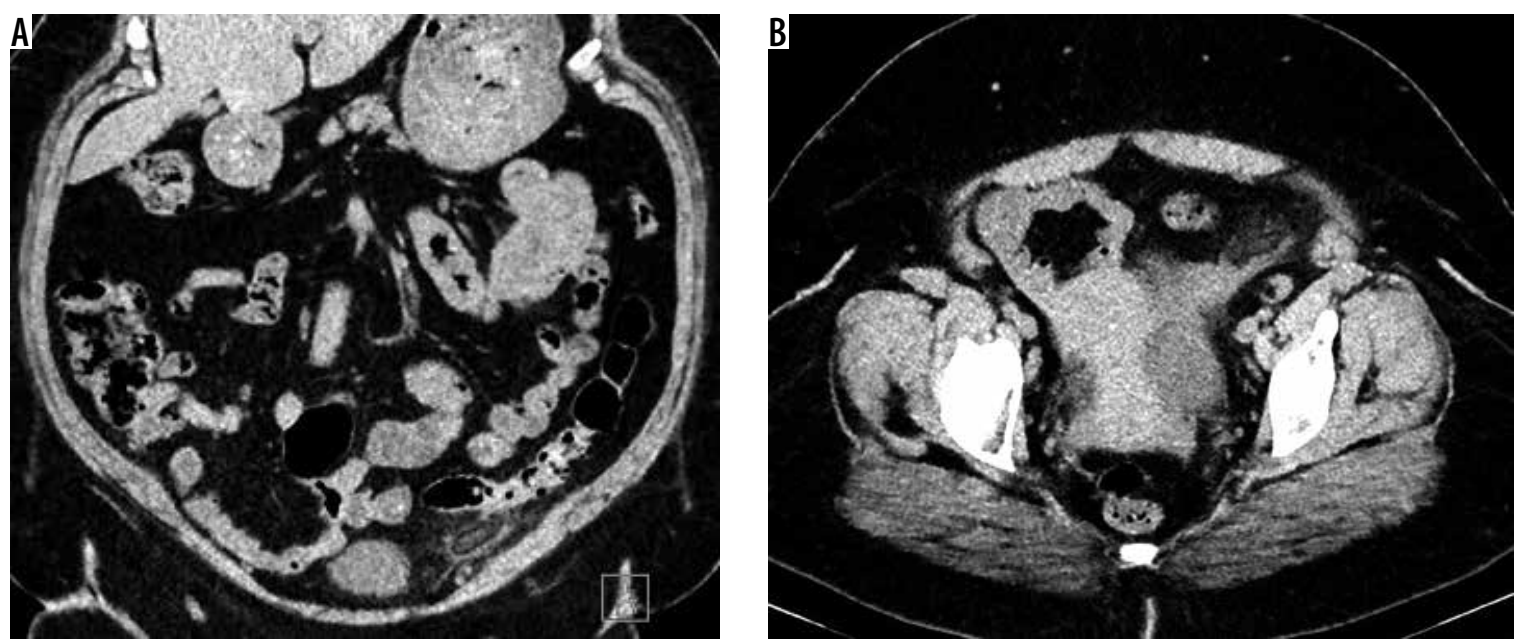

Figure 5. Epiploic appendagitis in a 25-year-old man with left lower quadrant pain. A) coronal and B) axial computed tomography images showed an oval area of fat attenuation and stranding adjacent to the left colon and surrounded by a ring of soft tissue $-\mathrm{a}$ finding indicative of epiploic appendagitis

CT is the best imaging modality upon which the diagnosis of omental infarction can be made. It shows an abnormal heterogeneous fat density lesion like a mass centred on the omentum, either triangular or oval in shape, and located between the colon and anterior abdominal wall. Moreover, if a whirled pattern strand is seen at the $\mathrm{CT}$, omental torsion is suggested to be the cause of omental infarction $[2,3,5]$.

Colonic diverticulitis is one of the causes of acute abdomen and should be excluded in cases of omental infarction. CT findings can exclude colonic diverticulitis by the absence of adjacent diverticulum, abscess, and bowel wall thickening. Although bowel wall thickening is a rare finding in omental infarction, it could be seen due to reactive bowel wall changes [10].

In the current study, MSCT showed an ill-defined heterogeneous density area of the omentum with surrounding fat stranding in 13 patients diagnosed as IOI, with no related colonic wall thickening or collections. Most of the lesions in eight patients (40\%) were between 5 and $7.5 \mathrm{~cm}$ and in five patients (25\%) they were less than $5 \mathrm{~cm}$, while no lesions were larger than $7.5 \mathrm{~cm}$.

In the other seven patients diagnosed by $\mathrm{CT}$ as acute EA, CT features showed oblong or oval shaped fat density lesion related to the colonic wall and surrounded by a peripheral hyperdense rim in addition to central hyperdense foci representing thrombosed vein. All the lesions were less than $5 \mathrm{~cm}$ in size. Similar findings were reported by Almeida et al. [9].

Regarding the clinical and provisional diagnosis of the patients included in the current study, five (25\%) were suspected to have acute cholecystitis; CT findings showed idiopathic omental infarction at the right upper abdomen with extension to the sub-hepatic and pre-cholecystic regions with no signs of acute cholecystitis.

Another eight patients (40\%) were suspected to have acute appendicitis with right lower quadrant pain; CT findings showed the IOI at the right lumbar region anterior to the colon with normal CT appearance of the appendix. Another four (20\%) patients were suspected to have diverticulitis and presented with acute pain at the left side; CT findings showed epiploic appendagitis with no related wall thickening and no detected colonic diverticulosis or related collection.

The last three patients (15\%) were suspected to have renal colic, and after a non-contrast CT of the urinary tract was done, the urinary tract was free, and a suspected area of abnormal fat density was seen related to the colon on the left side. A full-contrast CT study was done for full assessment, and CT findings showed signs of EA.

Kamaya et al. [3] reported that, in cases of acute EA, CT shows a hyperdense rim surrounding the fatty lesion and abutting the colonic wall in addition to central hyperdense foci seen in more than $50 \%$ of cases. On the other hand, in cases of acute IOI, the hyperdense rim if present is not abutting the colonic wall and no central hyperdense foci are seen. We obtained similar findings in the current study as in cases of acute omental infarction; the lesion was not abutting the colonic wall in 13 patients (65\%). Moreover, a continuous hyperdense rim around the fatty lesion was seen in three cases (15\%). All cases of acute epiploic appendagitis showed the classic appearance with central hyperdense foci in seven patients (35\%).

MSCT plays an important role in the diagnosis and management of acute omental infarction, to avoid unnecessary surgical intervention [11-13]. Management could be either conservative treatment with pain medication or laparoscopic resection of the inflamed omentum, especially if there are suspected complications [14,15].

Bowel obstruction due to adhesion or omental abscess may be seen as complications of omental infarction $[3,8]$. We did not face such cases in our study.

The lack of series studies on the primary omental infarction and the unknown risk of leaving an intra-abdomi- 
nal dead piece of omentum versus the risk of surgery lead to some controversy about the management [13]. CT imaging studies confirm the preoperative diagnosis, and the authors advise conservative treatment with antibiotics, analgesia, and hospitalisation if the patient is in a stable and uncomplicated condition. The aim is to avoid risk of postoperative adhesions. However, conservative management has a slow course and needs more time for abnormal resolution. It may last from four weeks to four months [13,14]. Although conservative management is applied in non-complicated cases, it is reported that it may end by complications like abscess formation, adhesions, or sepsis [14].

On the other hand, interventional management and resection of the inflamed omental segment is recommended by other authors relying on rapid recovery and better pain control. Also, the surgical management avoids risk of complications. Surgical management may be either laparotomy or laparoscopy, which has the advantage of visualisation of the other intra-abdominal organs, is minimally invasive, and the likelihood of missing the pathology is lower $[13,14]$.

According to the previously mentioned management plan, patients included in our study, who had omental infarction were managed by laparoscopic resection of the inflamed omentum, while conservative management was used for patients with epiploic appendagitis.

Hence, our retrospective study highlights and clarifies the CT imaging features of acute omental infarction.
The modality of choice is post-contrast MSCT, with the most common features of acute omental infarction seen as ill-defined, heterogeneous fat density lesion with surrounding inflammatory changes centred in the greater omentum. In cases of acute appendagitis, MSCT shows a central high attenuation focus within the fat or apposition of the fatty lesion related to the colonic wall. MSCT provides imaging features and confident diagnosis, which both help in the management plan decision of either conservative or laparoscopic resection.

\section{Conclusions}

Despite its low incidence, primary omental fat necrosis, either IOI or EA, should not be totally excluded in the differential diagnosis of acute abdomen.

MSCT is a basic imaging study that can diagnose primary omental fat necrosis and differentiate it from other causes of acute abdomen. Awareness of the MSCT imaging features of acute omental infarction is important for making the correct diagnosis and directing proper management.

\section{Disclosure}

The authors declared no conflicts of interest.

\section{References}

1. Bush P. A case of hemorrhage into the greater omentum. Lancet 1896; 147: 286.

2. Jeon YS, Lee JW, Cho SG. Is it from the mesentery or the omentum? MDCT features of various pathologic conditions in intraperitoneal fat planes. Surg Radiol Anat 2009; 31: 3-11.

3. Kamaya A, Federle MP, Desser TS. Imaging manifestations of abdominal fat necrosis and its mimics. Radiographics 2011;31: 20212034.

4. Singh AK, Gervais DA, Hahn PF, Sagar P, Mueller PR, Novelline RA. Acute epiploic appendagitis and its mimics. Radiographics 2005; 25 : 1521-1534

5. Singh AK, Gervais DA, Lee P, et al. Omental infarct: CT imaging features. Abdom Imaging 2006; 31: 549-554.

6. Barai KP, Knight BC. Diagnosis and management of idiopathic omental infarction: A case report. Int J Surg Case Rep 2011; 2: 138-140.

7. Tepeneua NF, Tarmannc R, Sinzigd M, Fasching G. Primary segmental omental infarction as a rare cause of acute abdominal pain in childhood. Journal of Pediatric Surgery Case Reports 2018; 28: 41-44.

8. Concanon ES, Hogan AM, Ryan RS, Khan W, Barry K. Primary omental infarction: a rare cause of acute abdominal pain. Clin Exp Med Sci 2013; 5: 233-240.
9. Almeida AT, Melão L, Viamonte B, Cunha R, Pereira JM. Epiploic appendagitis: an entity frequently unknown to clinicians: diagnostic imaging, pitfalls, and look-alikes. AJR Am J Roentgenol 2009; 193: 1243-1251.

10. Par TU, Oh JH, Chang IT, et al. Omental infarction: case series and review of the literature. J Emerg Med 2012; 42: 149-154.

11. Sand M, Gelos M, Bechara FG, et al. Epiploic appendagitis - clinical characteristics of an uncommon surgical diagnosis. BMC Surg 2007; 7: 11 .

12. AbdulAziz A, El Zalabany T, Al Sayed AR, Al Ansari A. Idiopathic omental infarction, diagnosed and managed laparoscopically: a case report. Case Reports in Surgery 2013; 2013: 193546.

13. Barai K, Knight B. Diagnosis and management of idiopathic omental infarction: a case report. Int J Surg Case Rep 2011: 2: 138-140.

14. Peirce C, Martin ST, Hyland JM. The use of minimally invasive surgery in the management of idiopathic omental torsion. The diagnostic and therapeutic role of laparoscopy. Int J Surg Case Rep 2011; 2: 125-127.

15. Awe JAA. Laparoscopic diagnosis and treatment of primary segmental omental torsion with infarction mimicking acute appendicitis: a rare cause of acute abdominal pain. Glo Adv Res J Med Med Sci 2014; 3: 54-58. 